\title{
Georreferenciamento como instrumento de gestão em unidade de saúde da família
}

\author{
Geoprocessing of data as a management tool in a family health unit
}

Geoprocesamiento de datos como herramienta de gestión en una unidad de salud de la familia

\author{
Erika Priscila Lisboa Müller', Márcia Regina Cubas', Laudelino Cordeiro Bastos" \\ 'Pontifícia Universidade Católica do Paraná. Programa de Pós-graduação em Tecnologia em Saúde. Curitiba, PR \\ "Universidade Federal Tecnológica do Paraná. Curitiba, PR
}

Submissão: 02/09/2009

Aprovação: 17/10/2010

\section{RESUMO}

Este estudo teve como objetivo geoprocessar dados de interesse para saúde numa Unidade de Saúde da Família. Foi necessário: levantar informações de conhecimento da equipe e outras fontes; organizar base de dados direcionada à base territorial para geoprocessar; e gerar mapas temáticos. É uma pesquisa descritiva-operacional Que utilizou o Sistema de Informação Geográfica (SIG). Os mapas temáticos permitiram visualizar o Quantitativo, a distribuição, a concentração e a incidência dos eventos, e possibilitaram correlações, dentre elas, a distribuição na área de gestantes de risco com o nascimento de crianças de risco, ancorando a análise do impacto das ações programadas. O mapeamento do território apóia o planejamento e a gestão dos serviços de saúde ajustados à realidade da população.

Descritores: Distribuição espacial da população; Programa Saúde da Família; Informática médica; Gestão em saúde.

\section{ABSTRACT}

This paper presents the results of geoprocessing data from a Family Health Unit in Curitiba-PR. A research dealt with both descriptive and operational aspects, using the Geographical Information System (GIS). It gathered available information from the Health Unit, identified which data is relevant for public health assessments, organized it in a database targeted for geoprocessing, and finally generated thematic maps. The paper focuses on cases of high-risk pregnancies and high-risk newborns. The thematic maps show the geographical distribution of the incidence and concentration of such cases, as well as correlations between them. Finally, the paper shows that georeferenced maps of public health issues support a better focused planning and management of health services offered to the local population.

Key words: Residence characteristics; Family Health Program; Medical informatics; Health management.

\section{RESUMEN}

El estudio tuvo como objetivo geoprocesar datos de interés para la salud en una unidad de salud (Curitiba, Paraná). Fue necesario levantar informaciones de conocimiento del equipo de la unidad, identificar las informaciones de interés para la salud, organizar una base de datos direccionados a la base de datos de la unidad para ser utilizada en un sistema de "geoprocesamiento" y generar mapas temáticos, una pesquisa descriptiva y operacional, Que utilizó el SIG. Los mapas temáticos posibilitaron visualizar: cuantitativo, distribución, concentración y incidencia de los eventos geocodificados como también posibilitaron analogías. La cartografía de la realidad del territorio es apropiado para apoyar la planificación y la gestión de los servicios de salud ofrecidos, ajustada a realidad de la población. Descriptores: Distribución del espacio de la población; Programa Salud de la familia; Informática médica; Gerencia en salud. 


\section{INTRODUÇÃO}

Nas últimas décadas, o rápido crescimento dos centros urbanos, aliado aos avanços tecnológicos e às mudanças estruturais globais, resultou em novas formas de produção e ocupação territorial, consolidando modificações nos hábitos da população e criando novos padrões de consumo( ${ }^{(1)}$ e, consequentemente, em desigualdades.

A união de estratégias de redução de desigualdades ambientais com as de promoção de justiça ambiental, tanto de cunho nacional Quanto local, promoveriam um enorme impacto na diminuição das heterogeneidades das condições de saúde ${ }^{(2)}$. Neste contexto, surge a necessidade de uma participação no âmbito das políticas de saúde pública para o estabelecimento de prioridades Que potencializem estas estratégias.

Alcançar a meta de promoção de justiça social, por meio da gestão territorial implica, necessariamente, na subdivisão do território nacional. $\mathrm{Na}$ área da saúde esta divisão é pautada na estratégia da descentralização, Que é um princípio constitucional do Sistema Único de Saúde (SUS) ${ }^{(3)}$.

Tal reorganização do território foi motivada pelo esforço em melhorar a efetividade na prestação da atenção à saúde, considerando uma base populacional definida ${ }^{(4)}$. Este espaço possui diferentes denominações, no entanto, o termo de uso corrente no Brasil é Distrito Sanitário - DS, cujo território deve ser apropriado visando o processo de planejamento e gestão(5) ${ }^{(5 u a ~ d i v i s a ̃ o ~ d e v e ~}$ considerar as características geográficas, populacionais, sociais, econômicas, culturais e epidemiológicas, de maneira Que o DS possua as características mais similares possíveis ${ }^{(6)}$. A área do DS podendo ser redividida, estabelecendo o espaço de ação de uma equipe de saúde, denominado de micro-área ${ }^{(7)}$.

O conhecimento do território de atuação de uma equipe de saúde é uma das estratégias utilizada para o diagnóstico e planejamento das ações em saúde do Programa de Saúde da Família - PSF, Que tem como um de seus princípios a adscrição de clientela em uma base territorial ${ }^{(8)}$.

Desta forma, o território passa a ter um papel fundamental e, em conjunto com o cadastramento das famílias vinculadas à Unidade de Saúde do PSF, se produz uma grande Quantidade a respeito da população Que reside no espaço, sendo necessária uma ferramenta mais adequada para armazená-los e visualizá-los no sentido de subsidiar a tomada de decisões no processo de planejamento em saúde. Esse complexo processo requer instrumentais Que transcendam a perspectiva burocrática gerencial, para permitir a consolidação da territorialização(9).

As temáticas território e tecnologia de informação têm sido consideradas pertinentes e atuais. Dentre os diversos modelos de sistemas de informação, o Sistema de Informação Geográfica SIG possui a capacidade de gerenciar dados complexos, pautados no componente geográfico do território. O SIG constitui-se numa estrutura de processamento eletrônico de dados Que permite a captura, armazenamento, manipulação, análise, demonstração e relatos de dados referenciados geograficamente ${ }^{(10)}$. Sendo assim, esse sistema poderia ser uma ferramenta apropriada para auxiliar os especialistas comprometidos com o processo de territorialização nos municípios.

As técnicas de geoprocessamento veem sendo utilizadas no planejamento, monitoramento e avaliação das ações de saúde, além de serem consideradas como ferramentas importantes de análise das relações entre o ambiente e eventos relacionados à saúde ${ }^{(1)}$. O geoprocessamento pode ser utilizado na análise dinâmica de difusão espacial das doenças e suas relações com o ambiente com alta resolução gráfica, na avaliação da situação de saúde de populações e na identificação de regiões e grupos sob alto risco de adoecer ${ }^{(12)}$.

O SIG possui grande capacidade para acessar e integrar diferentes níveis de informações (vetoriais, raster, de superfície e dados de campo e endereços), permite a apresentação e associação dos dados de diferentes formas (tabelas, gráficos e mapas temáticos) e possibilita o mapeamento, a exibição e a análise espacial dos dados relevantes para o processo de territorialização. Além disso, o sistema manipula os dados como os demais sistemas de informação e possui uma característica marcante de interligar atributos não espaciais a dados espaciais ${ }^{(13)}$.

A modelagem de um banco de dados territoriais, baseados em dados armazenados nas Unidades de Saúde é uma alternativa para cumprir a tendência atual de gerenciar um território espacial de forma contínua, com parâmetros variáveis no tempo e espaço ${ }^{(14)}$. Merece consideração o fato de Que o espaço local é dinâmico e a metodologia escolhida para trabalhá-lo deve ser aquela que permita sua reconstrução permanente ${ }^{(15)}$, convergindo com características do território digitalizado são as capacidades de representar a dinâmica do espaço e de simular o comportamento dos territórios ${ }^{(16)}$.

Diante deste contexto, a Questão norteadora a ser discutida neste artigo é: o SIG é uma ferramenta de gestão de dados territoriais adequada para auxiliar gestores e equipes de unidades de saúde da família na análise e planejamento de ações?

Para altercá-la, este manuscrito apresenta a utilização do SIG para organização de informações de interesse à saúde de uma unidade de saúde da família do município de Curitiba, capital do estado do Paraná. As etapas para operacionalizar este uso foram: a) levantar as informações de conhecimento da equipe; b) identificar, nos diferentes organismos públicos ou privados, as informações de interesse para a saúde; c) organizar uma base de dados em saúde direcionados à base territorial de uma unidade PSF para ser utilizada dentro de um sistema de geoprocessamento; e d) gerar os mapas temáticos para as informações obtidas.

\section{MÉTODO}

Este estudo caracteriza-se como uma pesquisa descritiva e operacional. Teve como cenário uma Unidade de Saúde do município de Curitiba-Paraná. A cidade ocupa um espaço geográfico de 432,17 Km² de área e sua população, segundo estimativa do Instituto Brasileiro de Geografia e Estatística - IBGE, para o ano de 2007, era de 1.818.948 habitantes.

No município, o PSF teve seu início, oficialmente, em 1995, com cinco unidades de saúde localizadas na região sul da cidade, no entanto, a prática da territorialização já se fazia presente na rotina das equipes de saúde da rede municipal. No modelo assistencial proposto pelo município, a Unidade de Saúde - US é responsável pela situação de saúde da população adscrita, sendo porta de entrada do usuário no sistema de saúde, viabilizando o acesso e o fluxo do usuário aos demais pontos de atenção ${ }^{(17)}$.

O estudo foi desenvolvido em três etapas: a primeira caracterizou 
o cenário de pesquisa por meio do levantamento das informações de conhecimento da equipe e da identificação dos dados de interesse para a saúde em diferentes organismos públicos ou privados. $\mathrm{Na}$ segunda houve a organização de uma base de dados de interesse à saúde para alimentar um sistema de geoprocessamento e a terceira tratou da geração de mapas temáticos a partir das informações obtidas.

Na primeira etapa do estudo foi aplicado um Questionário semiestruturado a seis pessoas ligadas a US, sendo: uma Agente Comunitária de Saúde, selecionada pelo critério de maior tempo de trabalho e conhecimento de todas as micro-áreas; a Autoridade Sanitária Local (chefia da US); os três Enfermeiros e a responsável pelo Centro Regional de Assistência Social da área da US. Além dos dados coletados na entrevista, agregaram-se os disponíveis em diferentes relatórios institucionais e no site do IBGE, sendo complementados por observação sistemática do território.

A caracterização do território ${ }^{(18)}$ foi operacionalizada classificando os dados para a coleta por: delimitações do espaço territorial; composição da equipe; diferentes áreas de espaços coletivos e de relevância geográfica; localização dos usuários de ações programadas em saúde, priorizando, neste momento: Criança, Mulher, Hipertenso/ Diabético e Saúde Mental; localização dos usuários portadores de doenças de notificação obrigatória; e outras informações de interesse à saúde. Os dados foram organizados em Quadros e codificados por semelhança, de forma a facilitar seu acesso.

Para a segunda etapa, foi necessário reorganizar os dados dos Quadros, em planilha eletrônica, de forma a relacioná-los aos respectivos endereços. Para cada informação a ser georreferenciada foram estabelecidos os campos relevantes, por exemplo, ao incluir um usuário do Programa de hipertensos, criaram-se campos para inserir a idade, sexo, classificação, existência de outra patologia crônica e adesão as atividades.

Para a última etapa, o geoprocessamento, foi utilizada uma base cartográfica digital, obtida junto ao Instituto de Pesquisa e Planejamento Urbano de Curitiba - IPPUC Que continha dados sobre: Altimetria, Equipamentos Urbanos, Infraestrutura, Limites Legais, Meio Ambiente e Trecho Rua. Esta etapa foi construída nos submomentos propostos pelo software Arcgis ${ }^{\circledR}$.

\section{RESULTADOS E DISCUSSÃO}

A US escolhida como base para a pesquisa era composta por Quatro Equipes de Saúde da Família, as quais atuam em Quatro microáreas. Muito embora o conceito de micro-área seja relacionado à homogeneidade do território e de sua população(7), a divisão estabelecida segue as normas do Ministério da Saúde para o PSF, Que infere o número de famílias atendidas, e não, necessariamente, a caracterização do território. Este fato faz com que cada micro-área seja subdivida novamente, respeitando, ao máximo, a semelhança das características da população e do território.

As equipes são compostas por um enfermeiro, um médico generalista residente, dois auxiliares de enfermagem, Quatro a seis agentes comunitários de saúde, um dentista, um a dois auxiliares de consultório dentário e um técnico de higiene dental.

Das cinquenta e sete informações de interesse para saúde que dariam corpus à caracterização do território da US, foram coletadas Quarenta e uma informações. Neste ponto, cabe uma discussão da importância e relevância deste hiato para o trabalho da equipe, exemplificando, pelo dado "emprego". Na entrevista foi possível levantar Que a principal ocupação dos moradores das áreas de risco era "carrinheiro" - coletor de material reciclável. Na capital paranaense, em 2005, existiam 2.769 carrinheiros $^{(19)}$, a partir deste número, houve a tentativa de estimar o Quantitativo na área da US, no entanto, essa parcela da população não declara a coleta de material reciclável como ocupação nos seus registros formais e possui uma alta mobilidade geográfica, não se estabelecendo em um único local da cidade ${ }^{(19)}$. Dado Que poderia facilmente ser monitorado pelo SIG, pois ao mapear estes indivíduos nos diferentes pontos da cidade, sua rotatividade poderia ser visualizada.

Outro dado importante diz respeito a crianças e adolescentes em situação de abandono, violência e evasão escolar. A dificuldade para obtenção destes dados se deve a alguns fatores: a rotatividade da população nas diferentes regiões de exclusão social da cidade; as famílias serem consideradas como "de risco" social ou biológico; o envolvimento com tráfico de drogas; e a presença de violência doméstica e comunitária. Uma das potencialidades do SIG é visualizar o mapeamento das famílias de risco Que são caracterizadas, além dos aspectos relativos ao território, pela baixa renda e por vivenciarem um difícil e complexo processo de enfrentamento de crises ${ }^{(20)}$. Verificar este fenômeno e sua mobilidade no território da cidade, auxiliaria no planejamento de ações direcionadas a estas famílias, Que, são expostas a interrupções em seu atendimento a cada mudança de cadastro nas diferentes unidades de saúde.

Ao executar a geocodificação foram verificadas duas situações Que interferiram na utilização do SIG. A primeira delas se refere a Que uma das micro-áreas não era lida pelo programa, pois a base digital de Curitiba, fornecida pelo IPPUC, do ano de 2002, não continha o arruamento da referida micro-área, por se tratar de área de ocupação irregular. Outra dizia respeito a dados inseridos nas tabelas Que não eram demonstrados no mapa digital, pois os nomes das ruas, bem como a numeração das casas estavam inconsistentes.

Estas inconsistências justificam-se por fatos oriundos da mobilidade do território: o nome da rua na base digital estava discriminado de forma diferente ao relacionado na listagem da US; a numeração das casas, referentes a Quadra digital, era diferente da real e algumas ruas possuírem seus nomes modificados pelos moradores. Para minimizá-las, a base cartográfica foi atualizada por meio de observação sistemática no território, o Que possibilitou conferir os nomes e os números de cada Quadra digitalizada; adequar os nomes das ruas em ambas as bases (a digital e a oriunda da coleta); incluir os números residenciais nas Quadras correspondentes; desenhar os seguimentos cartográficos Que não existiam na base anterior; e nomear e numerar os seguimentos cartográficos. Desta forma, a criação de mapas temáticos capazes de expressar a realidade territorial foi efetivada, revelando a complexidade do mapeamento do território.

Em relação às ações programadas da saúde da criança e mulher, devido a sua característica de sub-divisões por áreas de atenção, foi necessário priorizar os dados a serem utilizados, desta forma, foram georreferenciadas as crianças menores de um ano, as gestantes de risco, e as gestantes de baixo risco. O imperativo de geoprocessar os dados referentes às crianças de zero a um ano 


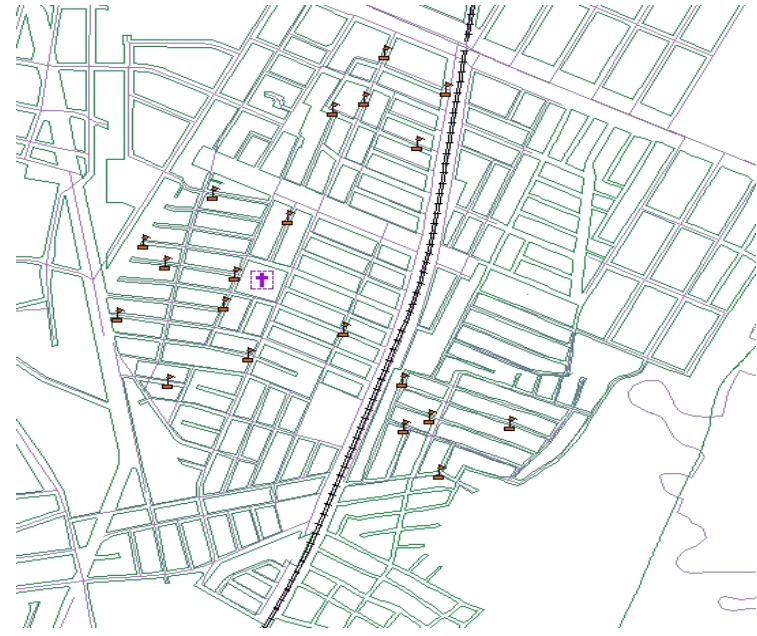

Figura 1. Geocodificação das crianças menores de um ano, consideradas de risco evolutivo social, no território da Unidade de Saúde.

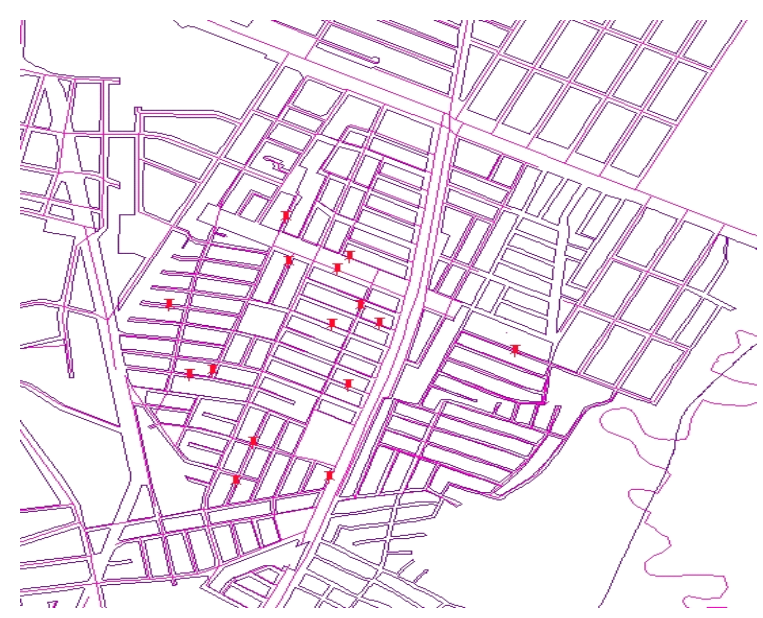

Figura 2. Geocodificação das gestantes de risco no território da Unidade de Saúde.

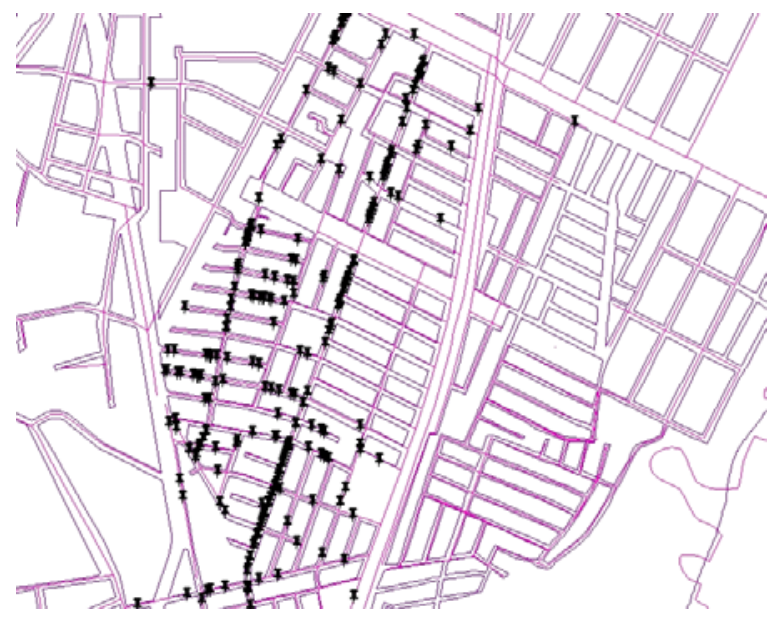

Figura 3. Geocodificação dos locais de comércio no território da Unidade de Saúde. direciona as ações a este público em especial, sendo a mortalidade infantil um indicador universal utilizado para monitoramento da Qualidade de vida e de saúde de uma população.

Outro ponto a ser discutido é o cruzamento do mapeamento dos menores de um ano com a cobertura vacinal, bem como com a incidência de doenças imunopreveníveis, favorecendo a efetividade de vigilância dos denominados eventos-sentinelas, Que tem como princípio a investigação do evento e a proposta de medidas de intervenção(21).

Em relação à assistência prestada pela US, os mapas temáticos permitem visualizar o Quantitativo, a distribuição/concentração, as áreas de maior incidência e os dados de cada evento geocodificado, referentes a cada ação programada. Numa descrição preliminar pode-se inferir Que uma grande concentração de menores de um ano, com Risco Evolutivo Social, provavelmente relacionados às condições do território em Que residem as famílias, observada pela visualização destes casos na área Que necessitou de atualização, ou seja, a Que não era oficialmente reconhecida pelo órgão oficial da Prefeitura, com condições ambientais vulneráveis (Figura 1).

Outro mapa exemplificado é o de geoprocessamento dos dados de gestantes cadastradas como de Risco (Figura 2). Este mapa permite, além da correlação citada no mapa das crianças, Que sejam feitas correlações entre as gestantes de risco e o nascimento de crianças de risco, permitindo a análise de impacto das ações do programa de gestantes.

A principal intenção de se mapear a realidade do território é o apoio ao planejamento dos serviços de saúde. A caracterização de áreas de lazer, consumo, espaços coletivos, educacionais, entre outros, podem indicar os diferentes acesso da população a bens e os riscos relacionados à falta de acesso. Este ponto pode ser cruzado com o geoprocessamento de outros fatores como morbidade e sexo, podendo potencializar análises de vulnerabilidades de gênero ${ }^{(22)}$.

O panorama destes dados pode ser exemplificado pelo mapa temático relativo aos comércios da área (Figura 3).

\section{CONSIDERAÇÕES FINAIS}

Ao aprofundar o conhecimento das distintas realidades das micro-áreas durante a apropriação do território, o propósito de um planejamento e gestão dos serviços de saúde oferecidos à população, ajustados à sua realidade, pode ser efetivado. Este trabalho auxilia no fortalecimento do plano de ações no primeiro nível de atenção à saúde, potencializando as diretrizes de adscrição da clientela e territorialização, e o princípio da descentralização.

Cabe ressaltar Que, pela diversidade brasileira, o uso da ferramenta de geoprocessamento de informações de interesse para saúde nem sempre é possível no espaço interno da US, pois é necessário computador e software adequados. Porém, é admissível coletar as informações e executar o geoprocessamento das mesmas em outro local, como foi realizado neste trabalho.

Os mapas temáticos, alimentados pela base de informações constituída e desenvolvidos com o software $\operatorname{ArcGIS}^{\circledR}$, podem auxiliar os gestores e as equipes de saúde na gestão dos dados territoriais e no planejamento de ações de saúde, por permitirem atualizações constantes dos dados, além de propiciar a manipulação e análise das informações geradas. 


\section{REFERÊNCIAS}

I. Augusto LS, Câmara VM, Carneiro FF, Câncio I, Gouveia N. Saúde e ambiente: uma reflexão da Associação Brasileira de Pós-Graduação em Saúde Coletiva - ABRASCO. Rev Bras Epidemiol 2003; 6(2):87-94.

2. Griffiths I. Mini-symposium: health and environmental sustainability. The convergence of public health and sustainable development. Pub Health 2006; 120(7):581-4.

3. Lucchese PTR. Descentralização do financiamento e gestão da assistência à saúde no Brasil: a implementação do Sistema Único de Saúde - retrospectiva 1990/1995. Plan Políticas Públicas 1996; 14: 75-156.

4. Paim IS. A reorganização das práticas em Distritos Sanitários. In: Mendes EV, organizador. Distrito sanitário: o processo social de mudança das práticas sanitárias do sistema único de saúde. São Paulo/Rio de Janeiro: Hucitec; 1995. p. 187-220.

5. Almeida ES, Castro CGJ, Vieira CAL. Distritos sanitários: concepção e organização. São Paulo: Faculdade de Saúde Pública da Universidade de São Paulo; 2002.

6. Bodstein R. Atenção básica na agenda da saúde. Ciência Saúde Coletiva 2002 7(3):40 I - 12.

7. Silva AMR, Oliveira MSM, Nunes EFPA, Torres ZF. A unidade básica de saúde e seu território. In: Andrade SM, Soares DA, Cordoni Junior L, organizadores. Bases da saúde coletiva. Londrina: Ed. UEL, 2001. p. 145-60.

8. Sousa MF, Hamann EM. Programa saúde da família no Brasil: uma agenda incompleta? [artigo na internet] 2007 Mar. [citado em: 2008 Out 02] Disponível em: http://www.abrasco.org.br/ cienciaesaudecoletiva/artigos/artigo_int.php?id_artigo $=44 \mathrm{I}$

9. Reis MAS, Fortuna CM, Oliveira CT, Durante MC. A organização do processo de trabalho em uma unidade de saúde da família: desafios para a mudança das práticas. Interface Comunic Saúde Educ 2007; I I (23):655-66.

10. Medronho RA. Geoprocessamento e Saúde: uma nova abordagem do espaço no processo saúde doença. Rio de Janeiro: FIOCRUZ/CICT/NECT; 1995.

II. Camara G, Monteiro AMV. Geocomputation techniques for spatial analisys: are they relevant to health data? Cad Saúde Pública 2001: 17(5): 1059-81.

12. Medronho RA, Werneck GL. Técnicas de análise espacial em Saúde. In: Medronho RA, Carvalho DM, Bloch KV, Luiz RR,
Werneck GL, editores. Epidemiologia. São Paulo: Atheneu; 2004. p.427-46.

13. Carvalho MS, Pina MF, Santos SM. Conceitos básicos de sistemas de informação geográfica e cartografia aplicados à saúde. Brasília: Organização Pan-Americana de Saúde; 2000.

14. Franco TB. Processos de trabalho e transição tecnológica na saúde: um olhar a partir do Sistema Cartão Nacional de Saúde [tese]. Campinas: Faculdade de Ciências Médicas, Universidade Estadual de Campinas; 2003.

15. Unglert CVS. Territorialização em Sistemas de Saúde. In: Mendes EV, organizador. Distrito sanitário: o processo social de mudança das práticas do Sistema Único de Saúde. São PauloRio de Janeiro: Hucitec-Abrasco; 1995. p.22 1-36.

16. Câmara G, Monteiro AM, Sposati A, Ramos FR, Koga D, Aguiar APD. Territórios digitais: as novas fronteiras do Brasil. Seminário Preparatório para a $3^{\circ}$ Conferência Nacional de Ciência e Tecnologia. [artigo na internet] 2007. Disponível em http:// www.cgee.org.br/cncti3/Documentos/Seminariosartigos/ Areasintnacional/DrGilberto\%20Camara.doc

17. Aguiar DS. "Saúde da família" no sistema único de saúde: um novo paradigma? [dissertação]. Rio de Janeiro (RJ): Escola Nacional de Saúde Pública, Fundação Oswaldo Cruz; 1998.

18. Mendes, EV organizador. Distrito sanitário: o processo social de mudança das práticas sanitárias do sistema único de saúde. São Paulo/Rio de laneiro: Hucitec/ABRASCO: 1995.

19. Brito MCC. A sustentabilidade do ambiente de uma comunidade por meio da promoção da saúde e da inclusão social digital estudo de caso: Vila Torres - Curitiba - PR [dissertação]. Curitiba: Centro de Ciências Exatas e Tecnológicas, Pontifícia Universidade Católica do Paraná; 2005.

20. Costa MS. Família em situação de risco: modelo de cuidado focalizando educação em saúde. Rev Gaúcha Enfermagem 2007; 28(I):45-5 I

21. Hartz ZMA, Champagne F, Leal MC, Contandriopoulos A. Mortalidade infantil "evitável" em duas cidades do Nordeste do Brasil: indicador de Qualidade do sistema local de saúde. Rev Saúde Pública 1996 30(4):310-8.

22. Pinheiro RS, Viacava F, Travassos C, Brito AS. Gênero, morbidade, acesso e utilização de serviços de saúde no Brasil. Ciência Saúde Coletiva 2002; 7(4): 687-707. 